\title{
A Framework for Automatic Tool Selection in Integrated CAPP for Sheet Metal Bending
}

\author{
T. H. M. Nguyen ${ }^{\mathrm{a}}$, J. R. Duflou ${ }^{\mathrm{b}}$ and J.-P. Kruth ${ }^{\mathrm{c}}$ \\ Department of Mechanical Engineering, Katholieke Universiteit Leuven, \\ Celestijnenlaan 300B, 3001-Leuven, Belgium \\ ${ }^{a}$ ThiHongMinh.Nguyen@mech.kuleuven.ac.be, \\ bJoost.Duflou@mech.kuleuven.ac.be, ‘Jean-Pierre.kruth@mech.kuleuven.ac.be
}

\section{Keywords: CAPP, sheet metal bending, tool selection}

\begin{abstract}
Sheet metal bending is a metal forming process, in which flat sheets are bent along straight bend lines in a specific bending sequence to form three-dimensional parts. A large number of tools with different characteristics can be used in this process. The task to choose the right tooling for a requested sheet metal part is however one of the bottle necks in process planning. An inefficient tool selection may result in failure of finding a feasible bending sequence. In previous work, methodologies for tool selection and optimization have been proposed. The presented paper describes a framework to implement these methodologies into a system that allows automatic tool selection in consistent consideration of bend sequencing. As a result, automated and optimized tool selection for sheet metal bending is achieved, as illustrated by performance test results for a robust software implementation.
\end{abstract}

\section{Introduction}

The capabilities of up-to-date CNC press-brakes allow producing more complex and compact parts in a rather short throughput time. Especially with the aid of a wide range of tools, part production has become much more efficient. Simultaneously, the task of preparing a feasible process plan has put an increasingly heavy load on process planners. Similar to other production processes, a lot of expertise is required to decide on production parameters, tool assignment, and operation sequencing. Consequently, time spent for this step becomes a more dominant factor for parts with high complexity and small batch size. Therefore, many efforts to automate this step have been spent over the past decade to solve the two crucial aspects of operation sequencing and tool selection.

Though forming two faces of an interlocking problem, tool assignment and operation sequencing for bending have never been dealt with simultaneously in any study. Since the part iteratively transforms during the process, causing a high risk of collisions, bend sequencing has attracted a lot of research $[1,2,3,4]$. As a result, computer aided solutions are currently available for automatic bend sequencing, including heuristic search techniques based on a rule set for collision avoidance $[2,3]$, and on tolerance specifications $[1,4]$. However, such approaches often rely on interactive tool selection or do not take tool selection into consideration. Consequently, if the tools are not well selected by process planners, feasible solutions cannot be assured.

In parallel, tool selection has been mentioned or partially dealt with by researchers in the field [5, $6,7,8,9]$. In consideration of the macro technological aspects, an expert system was built using LISP language to interactively aid process planners in choosing the right sheet metal process and tooling type $[5,6]$. In a more detailed perspective, existing automatic tool selection strategies often start from a predetermined bending sequence $[7,8,9]$. Such strategies frequently result in expensive constructions of specially tailored tools, which are needed to avoid obvious collisions that result from the chosen bend sequence [7, 8, 9, 10]. Traditionally, the approach chosen to eliminate the need for expert-based tool selection is a combinatorial search problem formulation for an open selection $[2,3,7]$. Under such regime, solutions for complex parts cannot be identified within a limited time span. 
In contrast to the previous researches, recent developments [11, 12, 13] have shown that an efficient selection system should consider not only the final part definition $[11,12]$, but also the intermediate shapes of the part [12] since in this unique process, the workpiece changes its shape and position continuously. Thus tools must not only be selected according to the envisaged product, but also be adjusted during the bend sequencing, if necessary. Moreover, optimization strategies [13] should be considered during tool selection in order to minimize the number of tools needed. An integrated system of automatic tool selection and bend sequencing is desirable only if it can take into account all these factors.

Complementary to the above-mentioned contributions of the current authors, this paper presents a framework for realizing such methodology by establishing a step-by-step procedure for automatic tool selection in an integrated CAPP system for sheet metal bending. The proposed system consists of a constraint generator that receives input from other modules that analyse the technological and geometric requirements. The constraints generated are used in the form of direct queries to be run against the tool database to retrieve feasible tools. Forming a crucial task, an optimization module minimizes the number of tools selected from the feasible tools provided. The described framework is based on a methodology making use of different techniques such as manufacturing knowledge processing, pattern matching, geometric reasoning, linear programming, and constraint generation for data query. The primary results for a series of benchmark parts, tested using the industrial software that implements the framework proposed, are provided subsequently to facilitate evaluation of the feasibility of the system.

\section{Tool Selection Methodology}

Tool selection methods for production processes typically consist of procedures to convert product specifications into selection criteria for related parameters in order to identify the relevant machines and tools. Moreover, where a minimal production resource is desired, optimization algorithms are usually applied. Both factors are collectively handled in the tool selection and optimization procedures for bent sheet metal products [11, 12, 13], and can be translated into the different considerations as explained below.

Feasibility Analysis. In order to provide a feasible production environment, the following considerations are taken into account for tool selection for sheet metal bending.

Technological Considerations. Similar to other production processes, tools selected for sheet metal bending should meet the technological constraints imposed by the part to be produced in order to assure technical feasibility and to provide the appropriate quality as determined by the design specifications. The relations between these two aspects are found scattered in literature, e.g. $[14,15]$. Based on these relations, tools are preselected under technological considerations in three subsequent steps [11].

The initial step is to select the bending technique, limited to air bending and bottoming in this study. The choice to be made depends on the accuracy, and the bend features required. The information of the bend angle accuracy is converted into the requirements for the bending techniques, and considerations for available in-process measuring and adaptive control equipment. The bend lines with special features are immediately provided with appropriate special tooling in this step.

The second step is to select the machine class, i.e. the range of setup length, tonnage, and gauging requirements. The force required to make the longest bend with the material and bending technique selected is calculated in order to specify the tonnage of the machine to be used. Bend lines with no parallel gauging solution imply selection of press-brakes with independent back gauges.

The third step is to select the tool class under the chosen bending techniques and machine classes. Decisions are made based on the required force for the bend features as well as their shape. This step takes as input various parameters of the part, such as the sheet thickness, material properties of the sheet, and geometric characteristics of the bend features to be performed, including the required internal radius, the bend angle, and the minimum bend-flange width. 
Geometric Considerations. In addition to complying with the technological considerations, the shape of the tool must correspond to the final as well as intermediate shapes of the envisaged part in order to avoid collision between the part and the tool. Therefore, tool selection can be divided into two phases. The first phase is called the preselection phase, which occurs before the bend sequencing to eliminate obvious collision-prone tool shapes based on the envisaged part shape. Preselection provides a favorable initiation for bend sequencing since it efficiently reduces the number of collisions encountered while searching for a collision-free bend sequence [12]. The second phase is called the refined selection phase, which selects the tools based on the collisions encountered by the part in its intermediate shapes during bend sequencing. If the tools are already preselected, refined selection only adjusts the preselected tools to suit the stricter conditions imposed by collisions encountered.

Though direct matching between available tool types and part shapes is not applicable due to the continuous shape transitions of the bent parts $[2,11,12]$, a generic approach capable of analysing the important three-dimensional part- and tool-features is desirable to quickly identify feasible tools that avoid collisions between the part and the tool [12]. Under geometric considerations, the shape features of the tools to be selected for bent sheet metal parts should correspond to the collision patterns that (likely) occur. For preselection, the collision patterns are detected based on a set of well-defined local details $[11,12]$ that can be singled out from the part based on their architectures. For refined selection, the collision patterns are directly identified by a collision detection module [16]. In case multiple collision patterns are foreseen per bend line, each of them is dealt with individually [12]. Subsequently, a constraint generator is triggered based on the obtained collision patterns input. The generation of such constraints is founded on a set of rules [12] linking the collision patterns to the specific geometric features of the tools. Examples of such rules are "choose tools with concave bodies for collisions along the bend line", or "choose tools with horn features, i.e. concave sides, for collisions at the side". Additionally, unsolvable collision cases, such as those with machine components [12], are immediately identified as an early feedback of infeasibility. This feedback terminates the current tool selection procedure and prompt for either an alternative sequence or a design revision. The range of values to be met by the shape features specified by the rules is calculated from the respective collision flange(s) of the collision pattern [11, 12]. Finally, the constraints are grouped per bend line to form a global constraint for tool selection for each bend. Under this procedure, all the geometric constraints for tool selection are recognized per bend.

Resource Optimization. The tooling solution selected based on the feasibility analysis discussed above can be considered as a starting point for the next task of determining the operation sequence, i.e. bend sequencing. However, the constraints generated based on these considerations act only as a filter to reject obviously incompatible tool sets and often leave multiple feasible tool solutions per bend line, which in turn may result in a large collection of tools. An increasing number of tools used means an expansion of the required space on the machine to mount all the tool stations. Such expansion often results in a higher setup time, a larger travelling distance between stations, and thus a lower efficiency of the operator [2]. In extreme situations, when the required station space is higher than what the available machines can accommodate, production must be split between more than one machine setup. These facts necessitate optimization methods that reduce or minimize the number of tools chosen for the respective bending operations. Three complementary strategies are proposed for optimization [13].

The first strategy tries to assign a tool to each bend so that the number of tools used is minimal, provided that all the possible tools are known for all bend lines. Under this approach, tool sharing between different bend lines is maximized. The implementation of this strategy makes use of a Set Covering Problem (SCP) formulation [17], which can be applied whenever multiple tools are simultaneously defined for single bend lines.

The second optimization strategy aims at maximizing the global preferences of tool utilization. This factor is calculated for each tool based on the production planning facts of the production company. In practice, tools preferred by either the company, the other parts in the same pool of jobs, or tools already used for other bend lines of the part have higher preferences to be chosen 
among other tools. This strategy results in a more intensive utilization of a group of highly preferred tools so that the global tool collection can be more compact. The changeover time for removing and mounting tool stations can be reduced accordingly.

The third strategy looks for possibilities to replace specific tools by similar ones that are already required by other bend lines in order to reduce the number of tool profiles in the final production setup. The similarities between the tools are judged by Euclidian distances between their vectors representing their intrinsic geometric features [13] in a multi-dimensional coordinate system. Tools with smaller distance to a specific tool are considered better candidates for replacement of that tool. Such replacement happens after a feasible tool assignment and bend sequence solution is found.

Constraint Concatenation. From the previous discussions on feasibility analysis, it can be seen that the technological and geometric considerations pose hard constraints for tool selection, since tools incompliant to these considerations are rejected. In contrast, production-planning considerations in the second strategy for optimization can be seen only as soft constraints that help to define the priority of selection between tools in order to achieve an optimized selection. Assembling all these scattered aspects requires a mathematical procedure to establish a useful representation of possible tool assignments per bend.

Hard Constraint. Firstly, the geometric constraints are combined with the technological constraints per bend line to generate a set of hard-constraints. Querying them on the tool database provides a set of tools satisfying this set of hard constraints. Since the bending tools are defined only by intersecting extruded polygons [16], i.e. $2 \frac{1}{2} \mathrm{D}$ objects, a few non-rejected tools left after the application of these constraints are then to be verified for actual three dimensional collisions with the part before being concluded as feasible. The tool feasibility verified is then presented in the form of a binary vector for that

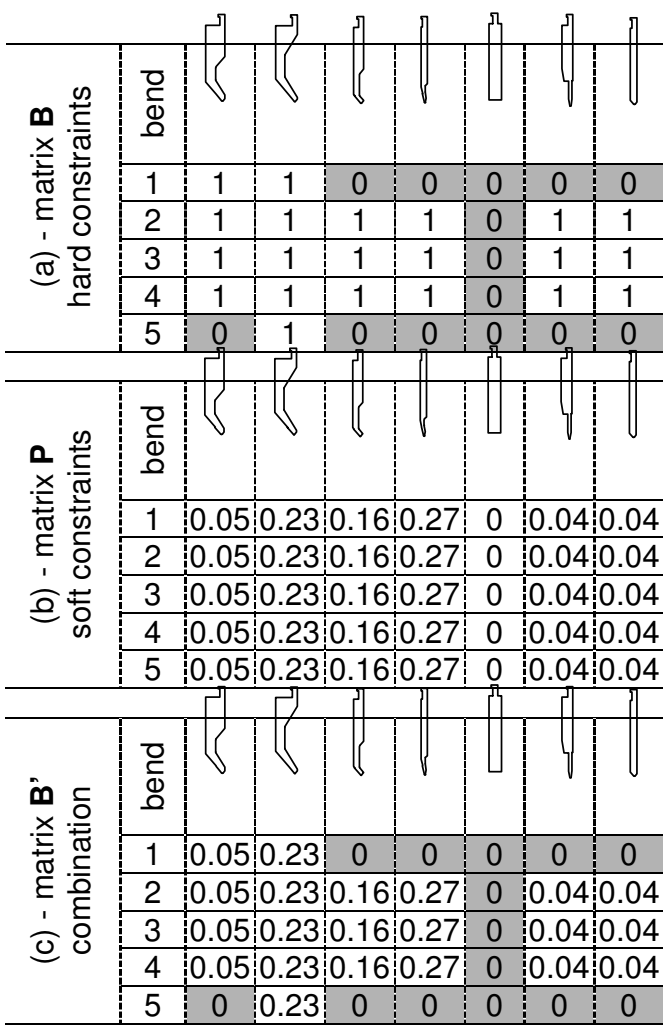

Fig. 1. Constraint concatenation bend line. An element $j$ having a value of one means tool $j$ is feasible for that bend line, while a value equals to zero indicates infeasibility for the corresponding bend-tool combination. All hard constraints of every bend line are then assembled in a binary matrix $\mathbf{B}$ where the rows correspond to the bend lines, and the columns - to the tools, as shown in Fig. 1.a. This matrix represents the filtering out of the impossible bend-tool combinations for the part under consideration.

Soft Constraint. Secondly, the preference factors are calculated and represented in the form of a matrix $\mathbf{P}$ also indexed according to the bend lines and the tools. This matrix shows the preference rating of all possible bend-tool combinations, as seen in Fig. 1.b. Such ratings are used as soft constraints for tool selection. In case other preferences are to be considered, they are kept in separate matrices.

Global Constraint. The two sets of constraints are then combined by taking a mask of the hard constraint matrix $\mathbf{B}$ on the preference matrix $\mathbf{P}$. This procedure provides a preselection matrix B' that reflects all the feasible bend-tool combinations with corresponding preferences. The sequence described is illustrated in Fig. 1.

Integrated Bend Sequencing and Tool Selection Procedure. An integration procedure including three phases is proposed in this section in order to provide a seamless integration of the tool selection methodology discussed above with the existing system of bend sequencing [2,3].

Phase 1: Preselection and Optimization. Utilizing a combination of hard and soft constraints, all feasible tools are recognized per bend line with respective preferences. This set of tools is called the preselection. In order to determine a single bend-tool assignment per bend line from this 
preselection, the second resource optimization strategy using an SCP model is applied. The output generated, being a feasible and optimized tool assignment per bend line, is a subset of the preselection. This initial tool selection serves as the starting point for the bend sequencing.

Phase 2: Bend Sequencing and Optimized Refined Selection. This phase consists of the three following tasks.

Bend Sequencing. This procedure seeks a feasible bend sequence basing on a Branch-and-Bound search using heuristics [3]. Starting from the completely folded part, the search advances to the node in the search tree representing the bend line that has the lowest penalty based on a set of precedence rules [2]. At each node, a three-dimensional collision check is performed. If the node is collision-free, then it is added to the solution sequence, i.e. the partially bent part is unfolded at this bend line.

Optimized Refined Selection. Otherwise, the collision pattern is analysed and additional constraints are generated to refine the preselection constraints for the current bend line [11, 12]. As a result, a set of feasible tools per bend line is proposed under this stricter condition. In order to achieve global optimization of tools, a single tool is picked out from this list of candidates based on its preference factor as discussed in the second optimization strategy for resource optimization.

Penalty Updating. Since using a new tool means that a new machine station must be allocated, an additional penalty is attached to each tool change. If no tool can be selected for the current collision situation, an infinite penalty is applied for that node. Thus all the paths starting from this node are pruned and no longer considered. If the new tool selected for the current bend line is already specified by the optimized preselection for other bend lines, no penalty is applied since no new station is needed. Otherwise, this penalty is calculated based on the preference of the tool discussed above in order to maximize the global preference of the tools selected.

Afterwards, the bend sequencing search advances again based on the total penalty of nodes, including the penalties from the set of heuristic rules and those from the selection of the new tool.

Phase 3: Optimization of the Solution. After a bend sequence is identified with a specific tool assignment, the corresponding process plan can still be modified in order to optimize the production resources. By applying the third optimization strategy discussed for resource optimization, likely candidates are identified and verified for collisions. As a result, when all feasible tools are known per bend line, the first optimization strategy can be applied again to obtain a compact tool set that also accommodates the bend sequence identified.

\section{A Framework for an Integrated System}

A framework for an automatic CAPP system for sheet metal bending is proposed in this section in order to support the methodology described above. The different considerations for a feasible and optimized tool selection are taken into account to establish functional modules. The system proposed consists of different modules, each of which supports a specific task or group of tasks, including interactions with the other modules. The task descriptions are explained in the next paragraphs.

Auxiliary Module 1: Database Management. A database rich with information stored in a predefined format is required to support direct queries generated from the Constraint Generator. Therefore, the information of machines and tools for bending is kept and maintained in a database located on a server and accessible for all process-planners. It contains information about the machines and their technological parameters, together with tool geometries, and tool management aspects such as availability.

Auxiliary Module 2: Part Preprocessing. To provide other modules with the relevant characteristics of the part under consideration, this module reads the CAD file of the part in order to extract (1) the geometry of the bend flanges, bend lines and bend angles, (2) the manufacturing parameters, such as thickness and bend radii, (3) the quality requirements of the part, (4) the batch production information, and also (5) to identify the bend features used in the part. These data are kept in the temporary memory of the process-planning workstation at each run. 
Module 1: Technological Analyzer. This module deals with the input for the three technological steps of tool selection as discussed in the section of technological considerations. The information extracted by the Part Preprocessing module related to the technological aspects is analyzed, grouped and then transferred to the Constraint Generator to form the respective queries on technological parameters.

Module 2: Local Detail Analyzer. The Local Detail Analyzer is responsible for determining the collision patterns from local detail analysis. The task is divided into three subsequent steps. At first, the topological abstraction of the part is constructed in the form of a network with nodes and arcs [2] based on the geometric information obtained from the Part Preprocessing module. Secondly, the local details residing in the part are identified based on this part abstraction. Thirdly, the potential collision flanges with the corresponding collision patterns are identified based on these local details. The result is finally passed to the Constraint Generator to form the query on tool shape features.

Module 3: Constraint Generator. The technological and geometric considerations that have been described for feasibility analysis are represented by a set of IF-THEN rules that is used by the Constraint Generator to generate the queries for tool selection. Based on the triggering of these rules, two groups of constraints are generated. The first group contains the technological constraints on bending technique, machine class, and technological parameters, generated from the values taken from the Technological Analyser. The second group contains the geometric constraints on the tool shape features, generated based on the coordinates of the collision area and collision patterns passed from either the Local Detail Analyser or the Bend Sequencing and Refined Selection module. As the result, a series of hard constraints is generated per bend line and then passed to the Constraint Concatenation module.

Module 4: Constraint Concatenation. All the constraints are combined in this module in three subsequent steps. Initially, the hard constraints are applied by taking the output of the Constraint Generator and passing the query to the tool database. The results are verified for collision before being stored in the feasibility matrix. Meanwhile, the soft constraints are applied by taking the preferences of bend-tool combinations from the Global Preference Estimator to generate the preference matrices. When both matrices are available, they are concatenated as previously described in the section of constraint concatenation. It could be noticed that since the hard constraints for each bend line are updated during the integrated bend sequencing and refined tool selection, the combined matrix is also continuously updated during the process planning of the part.

Module 5: Optimization. The different optimization strategies described for resource optimization are realized in this module by three submodules. Each of them is called at a specific stage of process planning to generate a feasible and optimized solution as described below.

The first submodule is an Optimizer for Multiple Bend Lines using an SCP model. Taking the output matrix from the Constraint Concatenation, it is activated in the first and third phase of the integrated bend sequencing and tool selection procedure described previously in the text. To enhance the convergence of the algorithm, the matrix is binarilized before being used as input. This procedure eliminates the feasible bend-tool combinations with preferences lower than a threshold value.

The second submodule is a Global Preference Estimator that provides the input for the generation of soft constraints. It calculates and applies the different preferences reflecting various levels of soft constraints. The input for this submodule is provided by recording the dominant tool sets in use within the company and possibly also from the Part Preprocessing module.

The third submodule is the Tool Similarity Analyzer. The similarity indices [13] between the preselected tools are calculated by this module. When considering the replacement of a tool, the similarity indices of other tools in comparison to that specific tool are called and ranked in order to find the most promising candidate for the replacement. The candidates are then verified for collisions at the bending stages in which the replacements of tools are proposed.

Module 6: Bend Sequencing and Optimized Refined Selection. To support the procedure described earlier in the text for the integration of automatic tool selection with bend sequencing, this 
module consists of three submodules. The first submodule is Bend Sequencing that uses an existing Branch-and-Bound search engine [2]. It can identify collision information to pass to the Optimized Refined Selection submodule. This second submodule can be activated by the first submodule to select a new feasible and optimized tool for the bend line by interacting with Modules 3, 4, and 5 . Then, the Penalty Calculator submodule is also activated to update the penalty value for the corresponding node in the Branch-and-Bound search tree and passes this value back to the first submodule. The Bend Sequencing continues with this updated penalty to look for a feasible solution.

\section{Experimental Implementation}

This framework has been implemented as a part of an evaluative version of a commercial software solution, however excluding the Optimization Module. A series of test parts has been processed on the platform of a PC Pentium M 1.4GHz, 512MRAM with Windows XP as OS. The performance of the system was recorded by the total processing time for finding feasible process plans, including automatic bend sequencing and refined tool selection. The automatic process planning is tested by several runs for each part, and the time recorded in seconds is averaged. It can be seen from Fig. 2 that for all the test parts, feasible bend sequences were found within less than one minute with the tools automatically selected according to the geometry of the part.

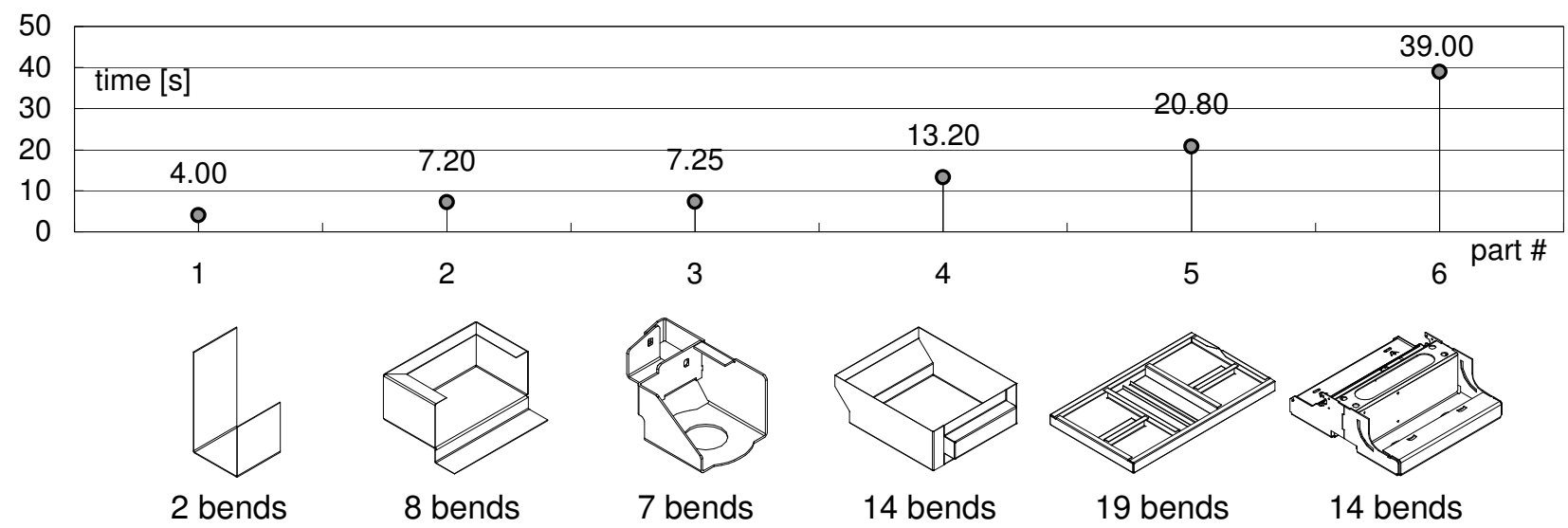

Fig. 2. Performance record for test parts

\section{Conclusions}

This paper describes a framework design for automatic tool selection in an integrated CAPP system for sheet metal bending. First of all, the procedures integrating the diverse considerations for tool selection, including feasibility and optimization analysis, were discussed. Based on these procedures, a systematic framework was proposed, consisting of functional modules that seamlessly work and interact in order to generate a feasible and well-optimized tool solution. It can be seen that in order to provide a fully automatic CAPP tool for bent sheet metal products, the two aspects of tool selection and bend sequencing should be considered simultaneously. The feasibility of such system is demonstrated by means of an experimental implementation. The software prototype is able to automatically generate feasible process plans for a number of test parts within a limited time interval.

\section{Acknowledgments}

The authors recognize the financial support from IWT-Vlaanderen (Instituut voor de Aanmoediging van Innovatie door Wetenschap en Technologie in Vlaanderen). 


\section{References}

[1] de Vin, L. J., de Vries, J. and Streppel, T., Process planning for small batch manufacturing of sheet metal parts, Int. J. Prod. Res., 38(17), 2000, 4273- 4283

[2] Duflou, J., Kruth, J.-P., Van Oudheusden, D., Algorithms for the Design Verification and Automatic Process Planning for Bent Sheet Metal Parts, CIRP Annals, 48(1), 1999, 405-408.

[3] Duflou, J. R., Collin, P., Kruth, J.-P., and Van Oudheusden, D., Automatic Process Planning for Bent Sheet Metal Parts: Implementation Aspects and Performance Evaluation, Proc. of 9th Int. Conf. on Sheet Metal, Leuven, 2001, ISBN 90-73802-78-4, 371-378.

[4] Shpitalni, M. and Radin, B., Critical Tolerance Oriented Process Planning in Sheet Metal Bending, Trans. of ASME J. of Mechanical Design, 121, 1999, 136-144.

[5] Singh, R. and Sekhon, G.S., An expert system for optimal selection of a press for a sheet metal operation, J. Materials Processing Technology, 86(1-3), 1999, 131-138.

[6] Singh, R. and Sekhon, G. S., An intelligent system for optimal selection of dies and tools for sheet metal operations, J. Engineering Manufacture, 216(B), 2002, 821-828

[7] Franke, V., Automation of Tool Planning for Bent Components, Proc. of 3rd Int. Conf. on Sheet Metal, Birmingham, 1995, ISBN 095276640 X, 35-44.

[8] Gupta, S.K, and Bourne, D.A., Sheet Metal Bending: Generating Shared Setups, ASME J. of Manufacturing Science and Engineering, 121, 1999, 689-694.

[9] Alva U., and Gupta, S. K., Automated Design of Sheet Metal Punches for Bending Multiple Parts in a Single Setup, Robo. and Comp. -Integ. Manuf., 17(1-2), 2001, 33-47.

[10]Franke, V., Backes, F. and Geiger, M., Automated Construction Methods for Press Brake Tools, Proc. of 4th Int. Conf. on Sheet Metal, Enschede, 1996, ISBN 90-365-08045, II, 427434.

[11]Duflou, J., Nguyen, T.H.M., Kruth, J.-P., Intelligent Tool Preselection - A Contribution To Automatic Process Planning For Sheet Metal Bending, Proc. of 4th Int. Symp. on Tools and Methods for Competitive Engineering, Lausanne, 2004, Millpress, ISBN 90-5966-018-8, 671682.

[12]Duflou, J., Nguyen, T.H.M., Kruth, J.-P., Geometric Reasoning For for Tool Selection in Sheet Metal Bending Operations, Proc. of 5th Int. Conf. on Integ. Design and Manuf. in Mech. Eng., Bath, 2004, ISBN 1-85790-129-0.

[13]Nguyen, T.H.M., Duflou, J. R., Kruth, J.-P. and Cattrysse, D., Tool Optimization for Sheet Metal Bending Operations, 4th CIRP Int. Sem. on Intel. Comp. in Manuf. Eng., Sorrento, 2004, CUES Fisciano Salerno, ISBN 88-87030-79-0, 257-262.

[14]Benson, S. D., Press Brake Technology, A Guide to Precision Sheet Metal Bending, Soc. of Manufacturing Engineers, 1997, Michigan, ISBN 0-87263-483-3.

[15] Serruys, W., Sheet metalworking: State of the Art, Chapter 5, Belgium, 2002, LVD Company NV, ISBN 90-807224-2-1.

[16]Duflou, J.R., Dung, H.T., and Kruth, J.-P., A Collision Detection Algorithm for the Verification of Sheet Metal Bending Set-Ups, Proc. of 6th Int. Conf. on Sheet Metal, Enschede, 1998, ISBN 90-365-111-35, Vol. II, 209-220.

[17]El-Darzi, E., Mitra, G., Graph Theoretic Relaxations of Set Covering and Set Partitioning Problems, Eur. J. of Operational Res., 87/15, 1995, 109-121. 
Sheet Metal 2005

doi:10.4028/www.scientific.net/AMR.6-8

A Framework for Automatic Tool Selection in Integrated CAPP for Sheet Metal Bending

doi:10.4028/www.scientific.net/AMR.6-8.287 